\title{
Sistematización de las Cadenas Nodales Linfáticas de la Pelvis Femenina
}

\author{
Systematization of the Linphonodes in the Female Pelvis \\ "Elías Regules; * Laura Borgno \& ${ }^{* * *}$ Varinia Scanniello
}

REGULES, E.; BORGNO, L. \& SCANNIELLO, V. Sistematización de las cadenas nodales linfáticas de la pelvis femenina. Int. J. Morphol., 23(4):353-362, 2005.

RESUMEN: Las investigaciones sobre el nodo centinela en cánceres del aparato genital femenino, vuelven indispensable el sistematizar los nodos linfáticos pélvicos así como el empleo de una nomenclatura única. La importancia del nodo centinela en el cáncer de cuello uterino es cada vez más evidente. Desde los puntos de vista anatómico y quirúrgico, se debe ser preciso al momento de asignarle una determinada topografía y vincularlo con una cadena nodal. Sólamente unificando criterios, se podrán homogeneizar las distintas series de estudios en búsqueda de resultados comunes. El objetivo del trabajo fue estudiar las cadenas nodales linfáticas de la pelvis y sistematizar la ubicación de sus nodos, como apoyo anatómico a la investigación del nodo centinela en el cáncer de cuello uterino.

Mediante abordajes por vía abdominal de 10 pelvis femeninas fueron disecadas las cadenas nodales pelvianas. Los grupos nodales se vincularon con un eje vascular iliaco, tanto arterial como venoso. Se compararon los hallazgos con los de 21 linfadenectomías pélvicas de operaciones de Wertheim-Meigs y con estudios histológicos de linfadenectomías pélvicas operatorias.

La cadena iliaca externa fue la más rica en nodos destacándose los nodos ínter ilíacos e infravenoso, junto con los nodos del pedículo obturador, considerados éstos como integrantes de la cadena iliaca externa medial. Menos frecuentemente se encontraron nodos pertenecientes a la cadena iliaca interna. En un $60 \%$ de los casos, se comprobó la existencia de nodos iliacos comunes mediales (grupo del promontorio), que no siempre son incluidos en la linfadenectomía.

La sistematización de las cadenas nodales linfáticas es la base para asignar la denominación correcta al nodo centinela, en el cáncer de cuello uterino. Los nodos más comúnmente encontrados y que tienen mayor proyección quirúrgica, son los nodos infravenoso, obturadores e inter iliacos.

PALABRAS CLAVE: Linfonodos pélvicos; Linfonodo centinela; Cáncer cérvicouterino; Sistematización.

\section{INTRODUCCIÓN}

El desarrollo de las distintas técnicas quirúrgicas para el tratamiento de los cánceres genitales, en particular para aquellos que tienen su origen en el útero, jerarquizan la importancia de la linfadenectomía regional, tornando indispensable el conocimiento de sus vías de drenaje linfático. La celulolinfadenectomía, no sólo es parte del tratamiento locoregional de la enfermedad, sino también representa una instancia fundamental en el diagnóstico, estadificación y pro- nóstico del cáncer. El estado de los nodos linfáticos regionales, libres o no de enfermedad, influencia sobre el tratamiento adyuvante, pronóstico y sobrevida.

Los vasos linfáticos provenientes de los órganos genitales internos se drenan esencialmente en linfocentros pelvianos y lumbares, situados en el tejido conjuntivo de los espacio sub y retro peritoneales, alrededor de los vasos

* Profesor Agregado del Departamento de Anatomía Humana Normal, Profesor Adjunto de Clínica Ginecotocológica "C", Facultad de Medicina. Universidad de la República Oriental del Uruguay, Montevideo, Uruguay.

** Asistente del Departamento de Anatomía Humana Normal, Asistente de Clínica Quirúrgica “3”. Facultad de Medicina. Universidad de la República Oriental del Uruguay, Montevideo, Uruguay.

*** Asistente del Departamento de Anatomía Humana Normal, Asistente del Departamento de Cirugía Plástica y Reparadora. Facultad de Medicina. Universidad de la República Oriental del Uruguay, Montevideo, Uruguay. 
iliacos, aorta y vena cava inferior. Originados en el tejido conjuntivo por capilares, estos vasos se anastomosan formando redes muy ricas dispuestas en varios planos, a partir de las cuales se originan colectores linfáticos. En el trayecto de dichos colectores se interponen nodos constituidos por tejido conjuntivo, tejido linfoide y vasos linfáticos. Si bien, habitualmente los colectores atraviesan uno o varios nodos antes de alcanzar los colectores terminales, en ocasiones pueden no hacerlo. La infiltración de dichos nodos por células cancerosas altera la circulación linfática normal posibilitando el establecimiento de una circulación retrógrada.

El conocimiento del desarrollo embriológico y de la disposición anatómica de las vías linfáticas de la pelvis, facilita la comprensión de las distintas técnicas quirúrgicas empleadas en el tratamiento del cáncer de cuello uterino. En el embrión, los primeros espacios linfáticos aparecen en la región cervical, donde se unen para formar los sacos linfáticos yugulares, de cuya porción caudal deriva el conducto torácico. En el abdomen, los primeros sacos linfáticos en reconocerse son el saco linfático retroperitoneal y los sacos linfáticos posteriores. El saco retroperitoneal dará origen a los colectores y nodos peri-cavo-aórticos; los sacos posteriores originarán los colectores y nodos ilíacos. La íntima relación en el desarrollo de las cadenas linfáticas retroperitoneales y pelvianas explicaría la persistencia, en ocasiones, de vías alternativas de drenaje, que desde las vísceras pélvicas alcanzan directamente nodos abdominales.

La posibilidad de realizar tratamientos quirúrgicos con mayor efectividad oncológica, pero a su vez menos agresivos, es una realidad buscada en forma incesante en oncología. Es importante reflexionar sobre las siguientes afirmaciones de Massi (1996) "Creemos que el gold standard de los oncólogos ginecólogos, debería ser brindar las máximas posibilidades de curación con el menor costo posible para la paciente, en términos de riesgo operatorio y secuelas post operatorias. Existe una clara necesidad de tener nuevos criterios para identificar pacientes con enfermedad de bajo riesgo, quienes pueden beneficiarse de un tratamiento quirúrgico menos agresivo, sin disminuir sus chances de curación."

La evaluación del compromiso metastático de los nodos linfáticos regionales, en pacientes con tumores sólidos en estadios precoces, constituye un importante factor pronóstico; sin embargo, la mayoría de ellos no poseen metástasis nodales al momento del diagnóstico (Leong,1998). De esta forma, la realización de vaciamientos nodales linfáticos como estrategia terapéutica con frecuencia es innecesaria.

En el tratamiento quirúrgico del cáncer de cuello uterino, la linfadenectomía es un pilar fundamental, pero conlleva importante morbilidad y complicaciones. Si se pudie- ra limitar la linfadenectomía con márgenes de seguridad, sin extirpar los nodos linfáticos no comprometidos, se disminuiría en forma significativa la morbimortalidad y complicaciones. El estudio del nodo centinela puede llegar a brindar información útil en este sentido. En algunos cánceres se ha comprobado que la linfa del tumor se drena primariamente a un determinado nodo linfático, desde donde se disemina en forma secundaria. La detección de este nodo, mediante métodos químicos o centellográficos, su exéresis y estudio histopatológico pueden evitar, en ausencia de metástasis, la linfadenectomía.

El nodo centinela es aquel con mayores posibilidades de recibir células metastásicas a partir del tumor primario (Cabanas, 1977), es decir, el primer nodo linfático tributario de un tumor primario (Morton et al., 1992). Actualmente, se acepta el concepto que es cualquier nodo o nodos que reciben drenaje linfático directamente de un tumor primario (Uren,1999), definición que tiene en cuenta la existencia de más de un nodo centinela y de nodos en tránsito. $\mathrm{Su}$ histología es representativa de la correspondiente al resto de los nodos linfáticos del territorio en riesgo, lo que permite la linfadenectomía selectiva, es decir realizar la linfadenectomía sólo en pacientes con compromiso metastático en el nodo centinela.

El mapeo linfático pre e intra operatorio, sumado al estudio extemporáneo del nodo centinela, seguido de linfadenectomía selectiva, resulta una estrategia revolucionaria en el manejo de los tumores sólidos. En oncoginecología, Benjamín \& Rubin (1995) sostienen que es una técnica promisoria en el mapeo linfático del cáncer de vulva y, más recientemente, Levenback et al. (1994) y Levenback (2001) aplican la técnica en el cáncer de cuello uterino y de endometrio. Posteriormente, aparecen las serie de Verheijen et al.(1999) y Dargent et al. (1999), quienes sostienen que solo en aquellas pacientes con biopsia de nodo centinela positiva para metástasis, estaría indicada la linfadenectomía pélvica bilateral.

Si bien son numerosas las descripciones de las cadenas nodales linfáticas de la pelvis, no existe unanimidad en cuanto a su sistematización ni se utiliza una nomenclatura común entre anatomistas, ginecólogos, imagenólogos y patólogos para designar sus nodos. Con el desarrollo de los estudios sobre nodo centinela se vuelve indispensable sistematizar los nodos linfáticos pélvicos, así como emplear una nomenclatura única. Desde los puntos de vista anatómico y quirúrgico, se debe ser preciso al momento de asignarle al nodo centinela una determinada topografía y vincularlo con una cadena nodal. Solo así, unificando criterios, se podrán homogeneizar las distintas series de estudios en la búsqueda de resultados comunes. (Figs. 1 y 2). 

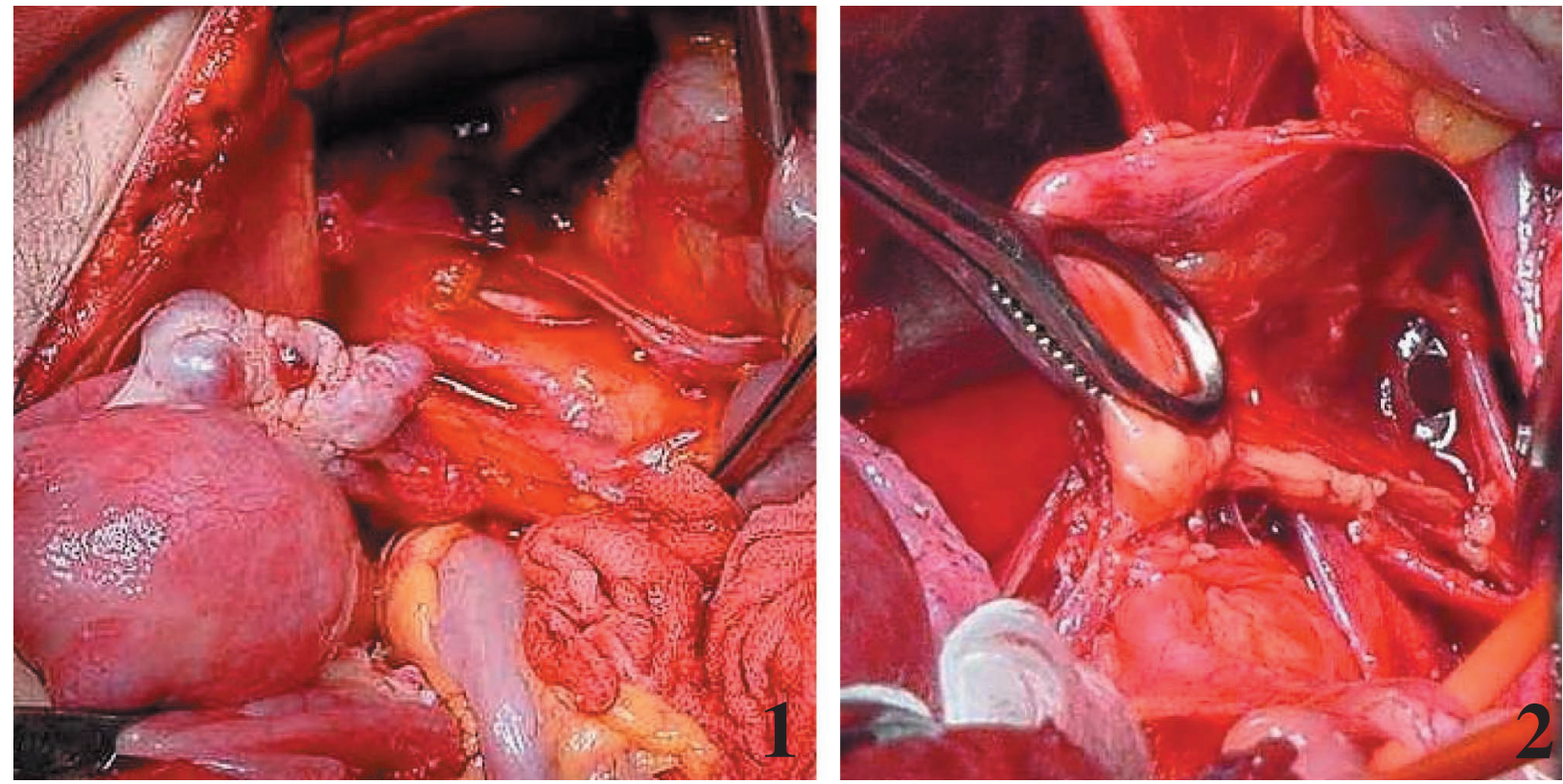

Fotos 1 y 2: Disección intra operatoria de los nodos de las cadenas iliacas externas.

El objetivo del trabajo fue estudiar las cadenas nodales linfáticas de la pelvis y sistematizar la ubicación de sus nodos, como apoyo anatómico a los estudios sobre nodo centinela en el cáncer de útero.

\section{MATERIAL Y MÉTODO}

Este es un estudio descriptivo, que se basa en la observación y disección cadavérica, comparando los hallazgos con aquellos obtenidos por los estudios histopatológicos de los tejidos de la celulolinfadenectomía pélvica de pacientes con cáncer de cuello uterino tratadas mediante cirugía.

Se utilizaron 10 pelvis femeninas del Departamento de Anatomía Humana Normal. Mediante abordaje por vía abdominal se disecaron las cadenas nodales pelvianas. Los grupos nodales se vincularon con un eje vascular iliaco, tanto arterial como venoso, precisando la ubicación de los nodos respecto a los mismos, así como el número de ellos.

Se compararon los hallazgos de las disecciones cadavéricas con los de 21 linfadenectomías quirúrgicas.

\section{RESULTADOS Y DISCUSIÓN}

Reseña anatómica: En 1740, William Hunter sostenía respecto a la función del sistema linfático: "Creo que he proba- do que los vasos linfáticos son los vasos absorbentes en todo el organismo, que son lo mismo que los vasos quilíferos y que éstos, junto con el conducto torácico, constituyen un sistema grande y general disperso en todo el organismo para la absorción; que este sistema sólo absorbe, no así las venas; que sirve para captar y transportar cualquier cosa que forma la sangre, o que se mezcla con esta última, desde la piel, desde el conducto intestinal y desde todas las cavidades internas o superficies" (Gervasoni et al., 2000). Esta es la primera correlación de los conocimientos anatómicos y funcionales del sistema linfático con la diseminación de enfermedades y tumores. Más de 100 años después, Virchow (1849) sostiene que los nodos linfáticos funcionan como barreras físicas a la diseminación de los cánceres.

Halsted (1894) en cáncer de mama y Moynihan (1908) en cáncer de colon, popularizan que la extirpación quirúrgica debe incluir el tumor, su órgano de asiento y las vías linfáticas de su diseminación; la intervención para tratar la enfermedad maligna no es sólo la cirugía de los propios órganos sino también la del sistema linfático.

La evolución de los conocimientos demostró que la extirpación radical de todos los nodos linfáticos regionales no siempre se asocia con beneficios, sino que muchas veces aumenta la morbimortalidad, llevando a pensar en una cirugía oncológica menos invasiva, de penetración corporal mínima.

Entre los estudio sobre el drenaje linfático de los órganos genitales femeninos, cabe citar los trabajos de Cúneo \& Marcille (1901), Poirier \& Cúneo (1902), Marcille (1903), 
Leveuf \& Godard (1923) y Leveuf (1931), Rouvière (1932), Dargent et al. (1963), Plentl \& Friedman (1971) y Dargent (1989).

Si bien todos los órganos de la pelvis son tributarios del linfocentro pelviano, en particular de los nodos iliacos externos, la linfa de los órganos genitales femeninos alcanza, además, los linfocentros situados en la ingle y retroperitoneo.

El linfocentro pelviano comprende los nodos linfáticos ilíacos o ilio-pelvianos, ubicados en la cavidad pélvica y escalonados a lo largo de los vasos iliacos a nivel del estrecho superior. La linfa visceral se drena hacia tres grupos nodales principales, a cada lado de la línea media: iliaco externo, iliaco interno e iliaco común.

Los nodos linfáticos iliacos externos conforman el principal grupo nodal, ubicado a nivel del estrecho superior, en relación con los vasos iliacos externos. En número de 8 a 10 , se agrupan a lo largo de estos vasos, formando tres cadenas: la cadena ilíaca externa lateral, supra-arterial; la cadena ilíaca externa intermedia o supra-venosa; y la cadena iliaca externa medial, infra-venosa.

La cadena iliaca externa lateral comprende de 2 a 4 nodos, situados a lo largo del borde lateral de la arteria. El más constante es el nodo lacunar lateral (nodo retro-crural externo), en relación con el origen de los vasos circunflejos iliacos profundos, que recibe colectores de los nodos inguinales profundos. Los restantes son poco frecuentes y cuando existen, se insinúan entre el músculo psoas y la arteria iliaca externa.

La cadena iliaca externa intermedia comprende 2 a 3 nodos inconstantes, situados por dentro de la arteria sobre la vena iliaca externa o entre ésta y la arteria. El más distal es el nodo lacunar intermedio (nodo retro-crural medio) inconstante; mientras que el más proximal es un nodo inter-iliaco, situado nivel de la bifurcación de la arteria iliaca común en externa e interna. Recibe colectores de los nodos linfáticos de la cadena iliaca externa medial, nodos linfáticos obturadores, nodos linfáticos inguinales y colectores directos del útero y vagina. Sus eferentes alcanzan los nodos linfáticos ilíacos comunes.

La cadena iliaca externa medial se sitúa por debajo y por dentro de la vena iliaca externa. Es la cadena más importante, compuesta por 3 nodos, 2 de ellos constantes y voluminosos. Para su visualización y disección es necesario elevar el tronco venoso. Se distingue un nodo inferior, nodo lacunar medial (nodo retro-crural interno), situado por detrás del anillo femoral, en relación con el nodo linfático de
Cloquet; un nodo medio, fusiforme, situado por debajo de la vena iliaca externa, inmediatamente por encima del nervio obturador (nodo de Leveuf y Godard), aplicado a la pared lateral de la pelvis cerca del orificio profundo del conducto del foramen obturador, cuyo compromiso metastático explica la neuralgia en dicho territorio nervioso; y un nodo superior, por delante del origen de la arteria iliaca interna. Esta cadena recibe la mayor parte de los colectores eferentes de los nodos linfáticos inguinales profundos y colectores de la vulva, así como también linfáticos de la vejiga, uréter pelviano, útero y vagina. Sus colectores eferentes alcanzan nodos linfáticos de la cadena iliaca externa intermedia, en especial el nodo inter-ilíaco.

Se describe además, un grupo de nodos linfáticos obturadores situados contra el pedículo obturador y el músculo obturador interno, cerca del conducto del foramen obturador. Reciben colectores de la vejiga, uréter, útero y vagina. Sus eferentes alcanzan las cadenas iliacas externas medial (nodo infra-venoso) e intermedia (nodo inter-ilíaco). Su exéresis se impone en toda linfadenectomía por cáncer genital pelviano.

Las cadenas iliacas externas homolaterales están unidas por anastomosis. Los colectores eferentes de los nodos más proximales alcanzan nodos linfáticos del grupo ilíaco común: desde la cadena lateral el nodo linfático distal de la cadena iliaca común lateral, desde la cadena medial el nodo linfático distal de la cadena iliaca común medial (nodo retrovascular) pasando por fuera de los vasos iliacos internos y desde la cadena intermedia ambas cadena iliacas comunes. Los nodos linfáticos de la cadena iliaca externa lateral sólo reciben colectores de los nodos inguinales, de allí que su exéresis sea de valor discutido en las célulo linfadenectomías por cáncer pelviano (Kamina, 2000; Cuneo \& Marcille).

Los nodos linfáticos iliacos internos se disponen en el intersticio de las ramas de la arteria iliaca interna. En número de 4 a 8 nodos, Cúneo \& Marcille, sitúan al más anterior de éstos entre los orígenes de las arterias umbilical y obturatriz, al más posterior sobre el tronco de la arteria glútea inferior (arteria isquiática) y pudenda interna y a los restantes entre los precedentes, en relación con las arterias uterina, vaginal, rectal media y glútea superior. Entre ellos se distinguen los nodos linfáticos glúteos y sacros.

Los nodos linfáticos glúteos superiores se sitúan entre la arteria glútea superior y la arteria iliaca interna; los nodos linfáticos glúteos inferiores rodean los orígenes de las arterias glútea inferior y rectal media. Reciben colectores del recto, regiones profundas del periné y región glútea. Los nodos linfáticos sacros se sitúan a lo largo de la arteria sacra lateral. En número de 2 o 3, se ubican por delante del 
segundo y tercer tronco nervioso sacro, su compromiso metastático explica las neuralgias en el territorio de los nervios ciáticos. Reciben colectores accesorios del recto y cuello uterino.

Gregoire (1962) jerarquizó las importantes relaciones de estos nodos linfáticos, al sostener que: "debemos admitir que la exéresis de los nodos linfáticos iliacos internos en una región tan pequeña, tan profunda, tan vascular, tan nerviosa y en donde muchas veces estos nodos se confunden con la grasa es una tarea bien difícil y muchas veces temeraria..."

Desde ellos se originan eferentes que alcanzan nodos linfáticos ilíacos externos y comunes. Los colectores eferentes hacia los nodos linfáticos ilíacos externos son los más numerosos. Pasan por fuera de la arteria iliaca interna para alcanzar los nodos proximales de la cadena iliaca externa medial o sus eferentes, o bien, transcurren por dentro de la arteria iliaca interna o umbilical para alcanzar los nodos de la cadena iliaca externa intermedia. Los colectores eferentes hacia los nodos iliacos comunes ascienden por detrás de los vasos iliacos internos para alcanzar la cadena intermedia o medial del grupo iliaco común homolateral.

Integrando este grupo de nodos linfáticos se describen nodos satélites de los vasos uterinos, entre los que se distinguen nodos yuxtauterinos en el trayecto de la arteria uterina próximos al entrecruzamiento con el uréter y nodos yuxtavaginales a lo largo de los bordes laterales de la vagina entre los que se mencionan uno situado por encima y detrás del fondo de saco lateral de la vagina (Lucas Championniére, 1870), uno situado por fuera del cuello uterino y un nodo útero-vaginal en la unión del útero y la vagina.

Las cadenas de nodos linfáticos ilíacos externos e internos drenan la linfa hacia nodos linfáticos ilíacos comunes homolaterales, situados entre el músculo psoas y la $5^{\mathrm{a}}$ vértebra lumbar, por delante del ala del hueso sacro (alerón sacro). Dispuestos por delante, a los lados y por detrás de los vasos iliacos comunes, estos nodos ocupan también la fosita ilio-lumbar, en cuyo fondo emerge el nervio obturador; reciben linfa de los nodos linfáticos ilíacos externos e internos, así como también colectores directos del útero y vagina que alcanzan el grupo nodal del promontorio. Los nodos linfáticos iliacos comunes se continúan sin límite de demarcación con los nodos linfáticos lumbo-aórticos, constituyéndose así en el principal grupo de drenaje de la linfa pelviana. Siguiendo a Cúneo \& Marcille se pueden distinguir a cada lado, 3 cadenas: lateral, intermedia y medial.

La cadena iliaca común lateral comprende de 1 a 3 nodos linfáticos que continúan la cadena iliaca externa late- ral. Situados a lo largo del borde lateral de la arteria iliaca común drenan hacia los nodos linfáticos latero-cavos y precavos a derecha. A izquierda drenan en los nodos linfáticos latero-aórticos izquierdos.

La cadena iliaca común intermedia se sitúa por detrás de los vasos iliacos comunes, entre el músculo psoas por fuera, la $5^{\text {a }}$ vértebra lumbar por dentro y el ala del hueso sacro por detrás. Ocupan la fosita ilio-lumbar cerrada por delante por los vasos iliacos comunes, la vena a la derecha y la arteria a la izquierda, por delante de la emergencia del nervio obturador y del tronco lumbo-sacro. Algunos nodos linfáticos de esta cadena se pueden situar por delante de las venas. Esta cadena drena en nodos linfáticos retro-cavos y retro-aórticos a derecha, mientras que a izquierda lo hacen en nodos linfáticos latero-aórticos izquierdos.

La cadena iliaca común medial ocupa, junto con la del lado opuesto, el ángulo entre las arterias iliacas comunes, en particular el espacio entre el borde medial de la arteria iliaca común derecha y la vena iliaca común izquierda. Los nodos de esta cadena están representados por el grupo nodal del promontorio constituido por la yuxtaposición por delante del promontorio y de la $5^{\mathrm{a}}$ vértebra lumbar de 2 a 4 nodos linfáticos infra y pre-venosos en relación con la vena ilíaca común izquierda, próximos al borde medial de la arteria iliaca común derecha. Esta cadena se drena por colectores anteriores y posteriores. Los colectores anteriores drenan hacia nodos linfáticos pre-aórticos a nivel del origen de la arteria mesentérica inferior, nodos linfáticos latero-cavos a derecha y nodos linfáticos latero-aórticos a izquierda pasando por delante del origen de las arterias iliacas comunes; los colectores posteriores drenan a nodos linfáticos retroaórticos.

Siguiendo a Kamina (2000), desde un punto de vista práctico y con criterio quirúrgico con relación al drenaje linfático visceral, se pueden considerar 2 niveles en el linfocentro pelviano:

El nivel I del linfocentro pelviano, limitado por los vasos iliacos externos e internos, comprende los nodos linfáticos de las cadenas iliacas externas, en especial intermedia con los nodos inter-iliacos y medial con los nodos infra-venoso y obturadores. Este nivel debe ser incluido en todas las linfadenectomías pélvicas.

El nivel II del linfocentro pelviano comprende los nodos linfáticos de las cadenas iliacas internas e iliacas comunes. Su exéresis se justifica en enfermedades más avanzadas.

Los troncos colectores del cuello uterino, en número de 2 a 8 , se desprenden a cada lado del sector supra-vaginal 
del borde lateral, formando en su origen un ovillo denominado plexo linfático peri-cervical. Dicho plexo es drenado por 3 pedículos eferentes que terminan en nodos linfáticos iliacos externos e internos, desde donde siguen distintas vías hacia grupos nodales proximales.

El pedículo iliaco externo (pre-ureteral), compuesto por 2 o 3 colectores, sigue a la arteria uterina en el parametrio lateral, pasando por encima del uréter, y cruza la arteria umbilical para alcanzar los nodos linfáticos de las cadenas ilíacas externas intermedia y medial. Según Reiffenstuhl (1957), en su mayoría terminan en el nodo linfático infravenoso y en nodos linfáticos inter-iliacos a nivel de la extremidad superior de la vena iliaca externa. Leveuf \& Godard han constatado que los colectores linfáticos del cuello uterino son tributarios con mayor frecuencia del nodo linfático infra-venoso de la cadena iliaca externa medial. Este pedículo anterior o pre-ureteral representa la principal vía de drenaje del cuello uterino, siendo su nodo principal el infra-venoso. El pedículo iliaco interno (retro-ureteral), compuesto por 1 a 3 colectores que oblicuos hacia atrás y afuera siguen a las venas uterinas principales, pasando por detrás y debajo del uréter, para alcanzar un nodo linfático de la cadena iliaca interna en la vecindad del origen de la arteria uterina. A veces un tronco colector supera el nivel de los nodos linfáticos iliacos internos y sigue hasta el grupo nodal del promontorio de las cadenas iliacas comunes, acompañando a la arteria iliaca común.

El pedículo posterior está representado por 2 a 4 colectores que se dirigen hacia atrás, contenidos en los ligamentos útero-sacros, hasta alcanzar nodos linfáticos sacros laterales y el grupo nodal del promontorio. Reiffenstuhl (1957) y Reiffenstuhl et al., (1998) describen en este pedículo, colectores que alcanzan nodos situados en el origen de las arterias glúteas superior e inferior. En particular, destaca la importancia de los nodos linfáticos glúteos inferiores en el drenaje de la linfa del cuello uterino, los que reciben colectores que transcurren por la porción baja del parametrio lateral. Dichos nodos no son accesibles a la exéresis quirúrgica, salvo con gran riesgo de lesión vascular o nerviosa. Su compromiso explicaría las denominadas recurrencias a nivel de la pared pélvica en la región de la espina isquiática. Schauta (1911) y Amreich (1924) coincidían en afirmar que "la linfadenectomía radical permite una extirpación sólo parcial de los nodos linfáticos regionales en el cáncer de cuello uterino”.

Estos distintos pedículos no se presentan con la misma frecuencia: el pedículo iliaco externo es constante, el pedículo iliaco interno existe, en dos tercios de los casos, terminando en la mitad de ellos en nodos linfáticos ilíacos internos, mientras que en la otra mitad lo hace en nodos linfáticos iliacos comunes (grupo nodal del promontorio), el pedículo posterior está presente sólo en un cuarto de los casos.

La disposición de los colectores linfáticos del cuello uterino es susceptible de presentar numerosas e importantes variaciones. A veces, existen grandes diferencias entre los resultados de las inyecciones linfográficas y las observaciones clínicas. Hartmann \& Lécene (1905) constataron en dos casos de cáncer de cuello uterino la existencia de metástasis en un nodo latero-aórtico derecho próximo al pedículo renal sin haber compromiso de nodos pélvicos. La diseminación hacia estos nodos se realiza, sea por medio de eferentes de los nodos linfáticos ilíacos o bien por anastomosis entre los colectores linfáticos del cuello y cuerpo uterino, en especial por un colector anastomótico latero-uterino que se continúa con el pedículo linfático principal del cuerpo del órgano.

Los troncos colectores del cuerpo uterino, en número variable, se agrupan en un pedículo principal y dos pedículos accesorios.

El pedículo principal o útero-ovárico: comprende de 4 a 6 troncos que, originados por debajo del cuerno uterino, acompañan al ramo ovárico de la arteria uterina en el ligamento útero-ovarico hasta el hilio de la gónada donde se reúnen con colectores del ovario y trompa. Contenidos en el ligamento lumbo-ovárico ascienden en el retro-peritoneo para terminar a derecha en nodos linfáticos latero-aórticos derechos, en especial en el nodo pre-cavo en la proximidad del origen de la vena cava inferior y a izquierda en nodos linfáticos latero-aórticos izquierdos y pre-aórticos próximos al origen de la arteria mesentérica inferior.

El pedículo accesorio iliaco externo: constituido por 1 o 2 colectores que, desde la parte media del borde lateral del útero, se dirigen transversalmente hacia fuera pasando por dentro y luego por encima de la arteria umbilical para alcanzar el nodo linfático inter-iliaco de la cadena iliaca externa intermedia a nivel de la bifurcación de la arteria iliaca primitiva.

El pedículo accesorio inguinal: constituido por un colector que, desde la inserción del ligamento redondo en el útero, trascurre contenido en dicho ligamento hasta alcanzar un nodo linfático inguinal superficial del grupo supero-medial.

Los linfáticos del cuerpo y cuello uterinos están ampliamente anastomosados entre sí a nivel de sus redes de origen, en la unión corpo-cervical y por intermedio del colector anastomótico latero-uterino a cada lado del órgano. A su vez, los linfáticos del cuello uterino se anastomosan con los de la vagina a nivel de sus redes de origen, la continuidad de los plexos peri cervical y peri vaginal explica las metástasis retrogradas en vagina de los cánceres de cuello uterino. 
Los distintos grupos nodales actúan como mecanismo de barrera y defensa frente a las células neoplásicas, su compromiso metastático es uno de los principales elementos pronósticos. La celulolinfadenectomía en el cáncer de cuello uterino clásicamente incluye la exéresis de los grupos nodales de las cadenas iliacas externa, interna y común en forma bilateral e incluso la resección de los nodos linfáticos lumboaórticos más distales. Si bien la presencia de metástasis nodales no cambia su estadificación, la presencia de micro y/o macro metástasis linfáticas modifica su pronóstico. El primer nivel nodal del drenaje linfático del cuello uterino corresponde a nodos de las cadenas iliacas externas intermedia y medial. A veces también deben ser incluidos en este nivel nodos linfáticos de la cadena iliaca interna y el grupo nodal del promontorio.

Hallazgos anatómicos: La forma con la cual se asigna la ubicación de los nodos en las cadenas linfáticas durante el acto operatorio, es variable según el cirujano y el centro hospitalario. Están aquellos que realizan la exéresis en bloque de todo el tejido celulolinfático de una cadena, mientras otros extirpan en forma individual cada nodo. Por otro lado la denominación que se les asigna no es uniforme ni se basa siempre en aspectos anatómicos.

Estos hechos motivaron que en este estudio se sistematizara la ubicación de los nodos linfáticos, en particular de las cadenas iliacas externas, tomando como elementos guías las arterias iliacas. Así, se consideraron como nodos de la cadena iliaca externa lateral a aquellos ubicados por fuera del eje medio de la arteria. Se tomaron como nodos de la cadena iliaca externa intermedia a aquellos ubicados entre la línea media de la arteria y el borde superior de la vena iliaca externa, considerando como nodos inter iliacos a los situados en el ángulo de la bifurcación arterial de la iliaca común, si bien podían estar aplicados a la cara medial de la vena iliaca externa.

Como nodos de la cadena iliaca externa medial fueron considerados aquellos que se ubicaban por dentro del borde superior de la vena, así como por debajo del tronco venoso hasta el nervio obturador. No se consideraron como integrantes de esta cadena aquellos que se enmarcaban en el ángulo arterial inter iliaco.

Si bien esta división puede ser arbitraria, nos permitió comparar los hallazgos con los resultados intra operatorios en la serie de nodos centinelas en la cual se identificaba el nodo o nodos radioactivos, se precisaba su ubicación y se realizaba su exéresis individual antes de proceder a la linfadenectomía completa.

La cadena iliaca externa fue la más rica en nodos dentro de los cuales se destacan los nodos inter iliacos e infravenoso, junto con los nodos del pedículo obturador, los que se consideran como integrantes de la cadena iliaca externa medial. Fig. 3. Con menor frecuencia fue posible encontrar nodos pertenecientes a la cadena iliaca interna. Fig. 4.
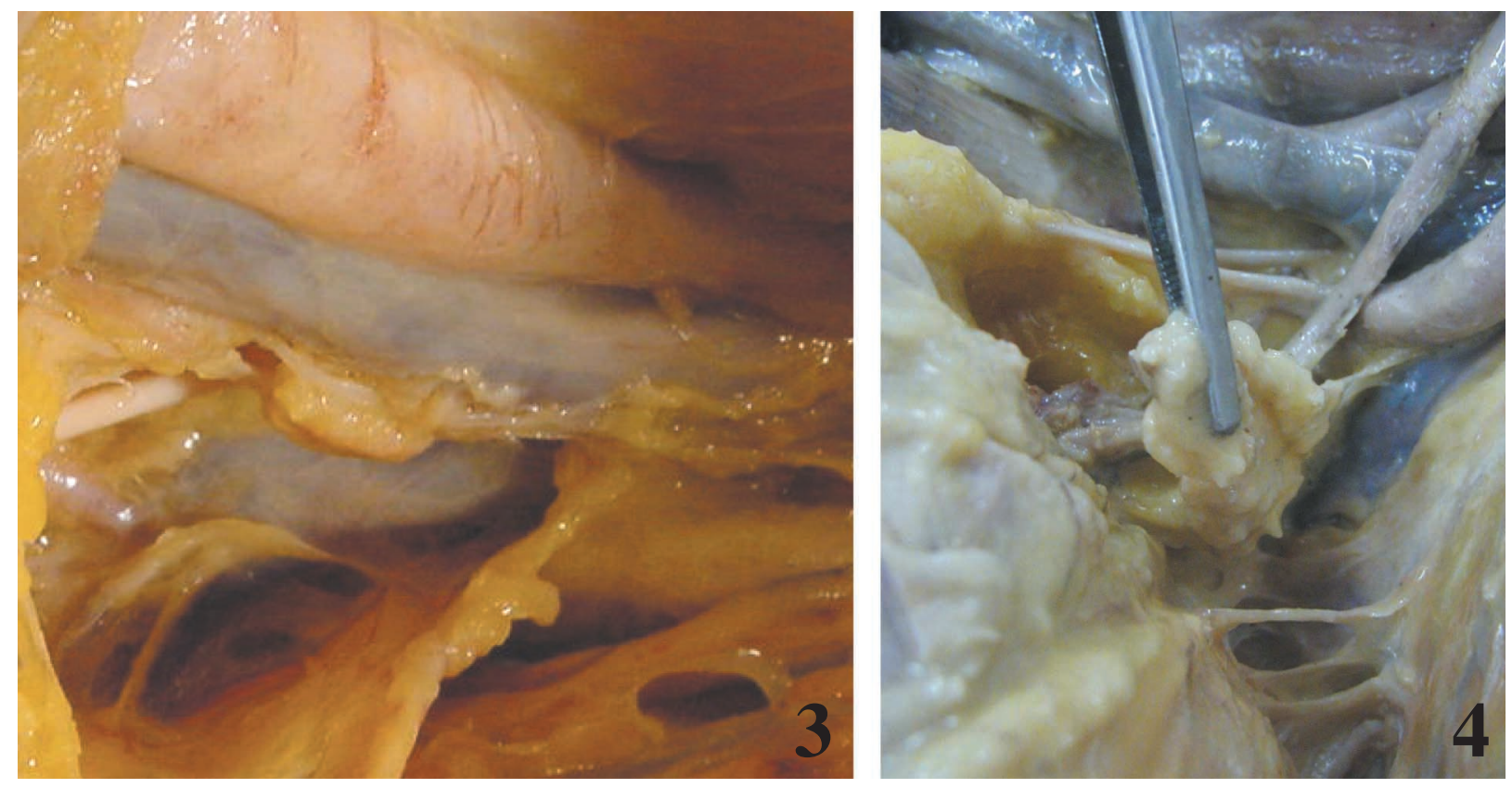

Fig. 3. Disección cadavérica de los nodos de las cadenas iliacas externas.

Fig. 4. Disección cadavérica de los nodos de la cadena iliaca interna. 
En un 60\% de los casos se comprobó la existencia de nodos iliacos comunes mediales (grupo del promontorio), que no siempre son incluidos en la linfadenectomía.

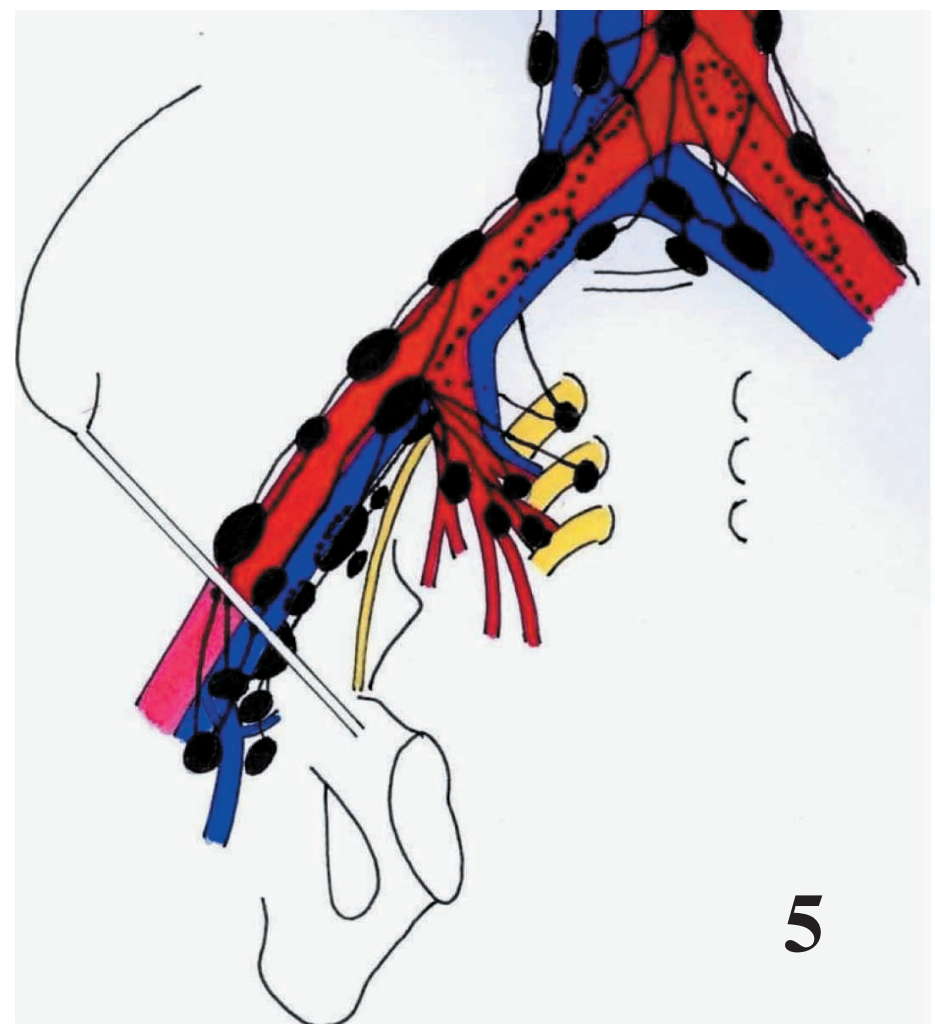

Existe una tendencia al predominio de las cadenas iliacas externas, en especial a las cadenas medial e intermedia, donde se ubicó con mayor frecuencia el nodo centinela en el cáncer de cuello uterino.

\section{CONCLUSIONES}

La sistematización de las cadenas nodales linfáticas es la base para asignar la denominación correcta al nodo centinela en el cáncer de cuello uterino. $\mathrm{Al}$ igual que en estudios clínicos, los nodos más comúnmente encontrados y que tienen mayor proyección quirúrgica, son los nodos infravenoso, obturadores e inter iliacos pertenecientes a las cadenas iliacas externas medial e intermedia. (Figs. 5 y 6 ).

La identificación del nodo centinela en el cáncer de útero puede ser la base para realizar la linfadenectomía pélvica sólo en casos necesarios, es decir, en aquellos con nodo centinela positivo para el diagnóstico de metástasis.

Figs. 5 y 6. Esquemas de las cadenas linfáticas de la pelvis y los pedículos de drenaje linfático del cuello uterino.
Comparación con hallazgos quirúrgicos: Los hallazgos de las disecciones cadavéricas se compararon con los hallazgos histopatológicos de 21 casos de cáncer de cuello uterino, estadios I b y II a, tratados mediante operación de Wertheim-Meigs (Arribeltz, 2003). En la citada serie se practicaron la centellografías pre e intraoperatoria, seguidas de identificación del nodo centinela que fue extirpado para su estudio histopatológico extemporáneo y en diferido. Luego, se procedió a realizar la linfadenectomía pélvica radical. Los nodos resecados fueron en promedio 21 (entre 6 y 49). De los nodos centinelas, 19 casos no tenían metástasis mientras que los 2 restantes sí las poseían.

El nodo centinela se localizó en la cadena iliaca externa en 14 casos (66,6\%), 8 a derecha y 6 a izquierda. De ellos, 8 correspondían a nodos infravenoso y/o obturadores $(38,1 \%)$ y 6 a nodos inter iliacos (28,5\%). En 3 casos (14,3\%) el nodo centinela pertenecía a la cadena iliaca interna, en 3 casos $(14,3 \%)$ a la cadena iliaca común y en 1 caso $(4,8 \%)$ era un nodo lumbo-aórtico. Es posible detectar más de un nodo centinela en el territorio marcado en el $28,5 \%$ de los casos.

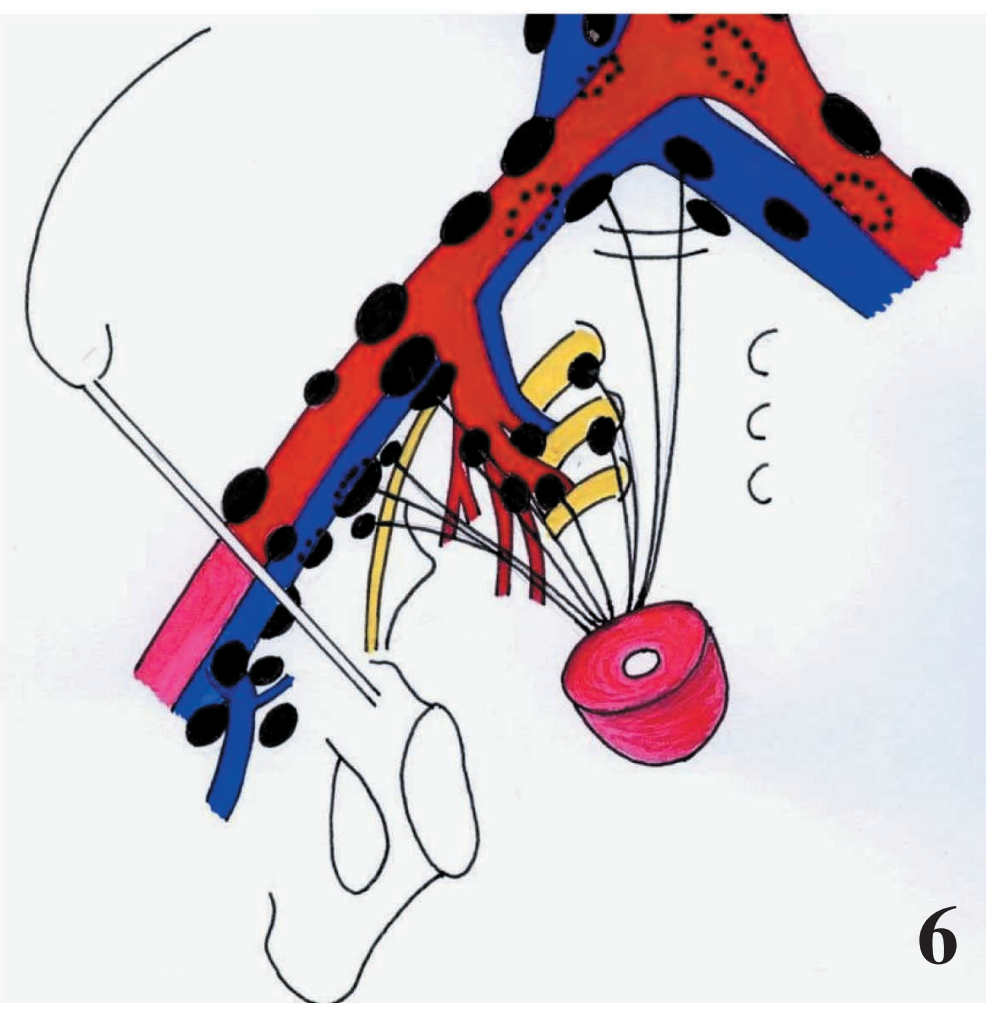


REGULES, E.; BORGNO, L. \& SCANNIELLO, V. Systematization of the linphonodes in the female pelvis. Int. J. Morphol., 23(4):353362, 2005.

SUMMARY: The investigation studies on the sentinel node in cancers of the feminine genital apparatus return indispensable systematizing the pelvic lymphatic nodes as well as the use of a unique nomenclature. The importance of the sentinel node in the cancer of uterine cervix is more and more evident. From the anatomical and surgical point of view we must be precise at the time of assigning certain topography and his relation with a nodal chain. Only unifying criteria, the different series from studies in search of common results will be able to be homogenized. The objetives to study the lymphatic nodes chains of pelvis and systematize the location of its nodes as an anatomical support to the investigation of the sentinel node in the cancer of uterine cervix.

Descriptive study based on anatomical dissections and histological studies of surgical pelvic lymphadenectomies. By abdominal dissection of 10 feminine pelvises the pelvic nodes chains were a boarded. The nodes groups tied with a iliac vascular axis, arterial as much venous. The findings with those of 21 pelvic linfadenectomías of operations of Wertheim-Meigs were compared.

The external iliac chain was the richest in nodes standing out the ínter iliac and infra venous nodes as well as the nodes of the obturator pedicule, considered like members of the medial external iliac chain. Less frequently were nodes pertaining to the internal iliac chain In a $60 \%$ of the cases the existence of medial common iliac nodes was verified (group of the promontory) that not always are including in the lymphadenectomy.

The systematization of the lymphatic nodes chains is the base to assign a correct denomination to the sentinel node in the cancer of uterine cervix. The nodes more commonly found and than have greater surgical projection are the infra venous, obturatores and inter iliac.

KEY WORDS: Lymphatic pelvic node; Sentinel node; Cervix carcinoma; Systematization.

\section{REFERENCIAS BIBLIOGRÁFICAS}

Amreich, A. L. Zur Anatomie und Technik der erweiterten vaginale Carcinoma colli uteri Operation. Arch. Gynäkal., 122:497, 1924.

Arribeltz, G.; Dabezies, L.; de Freitas, L.; Regules, E. et al. Investigación del "nodo centinela" en el cáncer de cuello uterino. Una propuesta nacional. Premio Nacional de Medicina 2003. Ministerio de Educación y Cultura. Academia Nacional de Medicina, Montevideo.

Benjamín, I. \& Rubin, S. C. Advances in surgery for gynecologic malignancies. Curr. Opin. Col., 7: 473-7, 1995.

Cabanas, R. M. An approach for the treatment of penile carcinoma. Cancer, 39: 456-66, 1977.

Cúneo, B. \& Marcille, M. Topographie des ganglions iliopelviens. Bull et Mém de la Soc. Anat de Paris., $6^{\circ}$ Serie. T III: 652-63,1901.

Dargent, D. \& Salvat, J. L'envahissement ganglionnaire pelvien. Medsi, Paris, 1989.

Dargent, D.; Martin, X. \& Mathevet, P Laparoscopic identification of the sentinel node in cervical cancer. In Proceedings and abstracts European J. of Nuclear Medi- cine. 1st International Congress on Sentinel Node in Diagnosis and Treatment of Cancer, Amsterdam, 1999.

Dargent, M.; Chassard, J. L. \& Dargent, D. La lymphographie iliopelvienne au lipiodol ultrafluide par voie pédieuse dans le cancer du col utérin. Semaine des hopitaux-annales de chirurgie., 17:1101-20, 1963.

Gervasoni, J. L. et al. Cita de William Hunter. En: Importancia biológica y clínica de la linfadenectomía. Clin. Quir. Nort., 6:1713-59, 2000.

Gregoire, R. Anatomie médico-chirurgicale de l'abdomen. La región lombaire et le petit bassin. Paris, Bailliére JB et fils, 1962.

Halsted, W. S. The results of the operations for the cure of cancer of the breast performed at the John Hopkins Hospital from June 1889 to January 1894. Johns Hopkins Hospital Rep ., 4:297-350, 1894.

Hartmann, H. \& Lecéne, P. Les propagations ganglionnaires dans le cancer du col de l'uterus. Annales de Gynécol et d'Obstetrique. $2^{a}$ Serie $T$ II :175-8,1905.

Kamina P. Anatomíe operatoire gynécologique et obstetricale. $1^{\mathrm{a}}$. éd. Paris, Maloine, 2000. 
Kamina, P. Anatomie gynécologique et obstétricale. $3^{\mathrm{a}}$ éd Paris, Maloine S.A., 1979.

Leong, S. P. L. The role of sentinel lymph nodes in human solid cancer. Principles and practice of Oncology. DeVita Jr., Hellman S, Rosenberg SA. Eds Philadelphia. Lippincott-Raven. 1998.

Leveuf, J. \& Godard, M. Les lymphatiques de l'utérus. Revue de Chirurgie. T LXI : 219-48. 1923.

Leveuf, J. L'envahissement des ganglions lymphatiques dans le cancer du col de l'utérus. Bull et Mém de la Soc. de Chirurgie:662-71, 1931.

Levenback, C.; Burke, T. W.; Gehershenson, D. M. et al. Intraoperative lymphatic mapping for vulvar cancer. Obstet Gynecol., 84:163-7, 1994.

Levenback, C. Intraoperative lymphatic mapping and sentinel node identification: gynecologic applications. Recent results. Cancer Res., 157:150-8, 2001.

Lucas-Championniére, J. Lymphatiques uterines et lymphangitis uterine. Thése, Paris, 1870.

Marcille M. Lymphatiques et ganglions ilio-pelviens. Tribune Médicale., 165-70, 1903.

Massi, G.; Savino, L. \& Susini, T. Vaginal hysterectomy versus abdominal hysterectomy for the treatment of stage I endometrial adenocarcinoma. Am. J. Obstet. Gynecol., 174 (4):132-26, 1996.

Morton, J. H.; Wen, D.R.; Wong, J. H. et al. Technical details of intraoperative lymphatic mapping for early stage melanoma. Arch Surg., 127:392-9, 1992.

Moynihan, B. G. The surgical treatment of cancer of the sigmoid flexure and rectum. Surg. Gynecol. Obstet., 6: 463-6, 1908.

Plentl, A. \& Friedman, E. A. Lymphatic system of the female genitalia. Philadelphia, W.B. Saunders, 1971.

Poirier, P. \& Cúneo, B. Les lymphatiques. In Traité d'Anatomie Humaine de Poirier et Charpy. Paris. T. II Fasc. 4, 1902.

Reiffenstuhl, G. The lymphatic system of the female genitalia. Urban e Schwarzzenberg, Munich, 1957.

Reiffenstuhl G, Platzer W, Knapstein PG. Operaciones por vía vaginal. Anatomía y técnica quirúrugica. Marban, Marban SL, 1998.

Rouvière H. Anatomie des lymphatiques de l'homme. Paris, Masson et Cie, 1932.

Schauta, F. Die erweiterte vaginale Operation beim Carcinoma colli uteri aufgrund zehnjähriger. Erfahrung. Mschr Geburtsh Gynäkal., 33:680, 1911.

Uren, R. Lymphatic drainage of the skin and breast. Locating the sentinel nodes. Uren, R. F.; Thompson, J.F. \& Howman-Giles, R. B. Eds Amsterdam, Harwood Academic Publishers, 1999.

Verheijen, R.; Pijpers, R.; Wust, M.; et al. Sentinel node detection in cervical carcinoma: A pilot study. Eur. J. Nucl. Med., 1st International Congress on Sentinel Node in Diagnosis and Treatment of Cancer, Amsterdam 1999.

Virchow, R. Zur diagnose der krebse in interleibe. Die Medinische Reform., 45: 248, 1849.

\author{
Dirección para correspondencia: \\ Dr. Elias Regules \\ Priamo 1495 \\ CP 11400 \\ Monterideo \\ URUGนAY \\ Email:eliastegules@yahoo.com.ar \\ Recibido : 04-08-2005 \\ Aceptado: 28-10-2005
}

